Section II. Systems and programs

\title{
PDPS: A Pulmonary Data Processing System for assessment of gas exchange properties by multiple gas wash-out
}

\author{
P.E.M. Huygen ${ }^{1}$, B.W.A. Feenstra ${ }^{1, *}$, E. Hoorn ${ }^{2}$, J.R.C. Jansen ${ }^{2}$ and A. Zwart ${ }^{3}$ \\ ' Department of Surgery, University Hospital, Rotterdam, The Netherlands ${ }^{2}$ Department of Pulmonary Diseases, Erasmus University, \\ Rotterdam, The Netherlands and ${ }^{3}$ TNO Toxicology and Nutrition Institute, Zeist, The Netherlands
}

\begin{abstract}
A data acquisition and processing system for the analysis of inert gas wash-out tests is described. The described system is in clinical use on spontaneously breathing patients as well as on mechanically ventilated Intensive Care patients. It combines several analysis techniques with an aim to provide a deeper insight into the nature of existing ventilation inhomogeneity than the individual techniques alone can provide. The signals measured are the respiratory flow, the fractions of one or two indicator gases washing out and the fractions of the metabolic gases oxygen and carbon dioxide. Analysis of these signals provides information concerning the end-expiratory lung-volume, distribution of ventilation and perfusion in the lung, the role of diffusion in the ventilation process and the metabolic oxygen uptake and $\mathrm{CO}_{2}$ release. This article describes the algorithms used and the results that are presented.
\end{abstract}

Ventilation inhomogeneity; Functional residual capacity; Lung volume; Multiple breath wash-out; Pulmonary gas mixing; Pulmonary gas exchange; Ventilation distribution

\section{Introduction}

Lung diseases like chronic obstructive pulmonary disease, emphysema, fibrosis or bronchus

\footnotetext{
* Present address: Medical Center Leeuwarden, Leeuwarden, The Netherlands.

Symbols and abbreviations: FRC, Functional Residual Capacity: gas-volume at the end of normal expiration; $V_{T}$, tidal volume; $V_{\mathrm{D}}$, dead space volume; $\dot{V}$, respiratory flow; $F_{1}$, inspiratory indicator gas-fraction before the beginning of the wash-out; $F_{2}$, inspiratory indicator gas-fraction after the beginning of the wash-out; $F_{\mathrm{l}}$, inspiratory gas-fraction; $F_{\mathrm{E}}$, end-expiratory gas-fraction; $F_{M}$, flow-weighted mean expiratory gas-fraction; $F_{\mathrm{A}}$, mean-alveolar gas-fraction at the end of an expiration; $t_{b}$, time of the start of an expiration; $t_{e}$, time of end of an expiration (start of next inspiration); $B$, Bohr dead space fraction; $R$, gas-exchange ratio.

Correspondence: P.E.M. Huygen, Department of Surgery, University Hospital Rotterdam, Dr. Molewaterplein 40, 3015 GD Rotterdam, The Netherlands.
}

carcinomata go with morphological and functional changes of the lung (for instance due to changes of the elasticity of the lung tissue or constriction of the airways). For the assessment of these changes and of the impairment of the gas-exchange process several lung-function tests are available. These tests are often aimed at measurement of lung volumes (e.g., the gasvolume in the lung at the end of a normal expiration (Functional Residual Capacity, FRC), or the maximum volume of air that can be inspired or expired) and the estimation of the increase of resistance to air-flow, e.g., measurement of the maximum volume of air that can be in- or expired within $1 \mathrm{~s}$. Most of the conventional lung-function tests require that the patient perform certain exercises like deep in- or expiration. This has several disadvantages: (1) not all patients are capable or willing to perform such exercises (this is especially true for young children); (2) the results 
of the tests depend on the collaboration and motivation of the patient and the skills of the laboratory assistant conducting the test; (3) the tests can not be performed during mechanical ventilation, when it is especially important to gain insight into the ventilation process in order to optimise it; (4) the tests provide insight into the abilities of the lung, but not into its performance during normal breathing.

The multiple-breath indicator gas wash-out test can be performed without active collaboration of the patient, and can reveal detailed information about the ventilation process. In such a test the subject inspires from a gas mixture in which the fractions of one or more insoluble, inert indicator gases change step-wise from a constant original value $F_{1}$ to a new (constant) value $F_{2}$. During the following breath-cycles, while the indicator gas fractions in the lung are altering, the inspiratory and expiratory flow as well as the gas fractions of the inspiratory and expiratory gas mixture are measured continuously. Usually wash-out tests are employed to measure the end-expiratory volume of the lung or to obtain a crude index of the inhomogeneity of the distribution of the inspired gas into the lung [1,2]. In some applications, the decay of the indicator gas-fraction is decomposed as the sum of a discrete number [3-5] or a continuous distribution [6-9] of mono-exponential curves representing regions with different ventilation-to-volume ratios. The software package described in this paper (PDPS, Pulmonary Data Processing System), however is aimed at providing a deeper insight into the ventilation process itself, the role that diffusion in the gas phase plays in ventilation $[10,11]$ and distribution of the ventilation/perfusion ratio. Therefore, it features multiple-gas wash-out (combining the wash-out of the gases helium and $\mathrm{SF}_{6}$, of which the diffusion coefficients differ by a factor 6), and measurement of the fractions of the metabolic gases oxygen and carbon dioxide.

Using the signals obtained, existing ventilation inhomogeneity can be characterised as the result of either (synchronous or asynchronous) emptying of parallel units with different specific ventilation ratios or diffusion-dependent inhomogeneity in the end of the bronchial tree (the acini) [10-13].
Comparison of the fractions of the inert gases washing out with the fractions of the metabolic gases oxygen and carbon dioxide can reveal whether the inhomogeneity in the ventilation is compensated by the alveolar perfusion or not. Finally, the metabolic rate can be calculated.

The advantage of PDPs is not the application of novel techniques, but the utilisation of the possibility that the mass spectrometer provides to measure several gases in the in- and expired air simultaneously. Multiple-breath wash-out and single-breath expirograms and dead space analysis have been used by Fowler [14], and extended by Tsunoda [15] and Lewis [8], whereas the gasexchange ratio analysis has been exploited by Serra [16] and Rahn [17]. Due to our combinatory approach, typical ventilation patterns, characteristic for lung diseases like obstructive pulmonary disease, emphysema or cystic fibrosis can be recognised. These individual methods alone are insufficient to make this discrimination.

This paper describes the operation of the program, the algorithms used in the analysis of the measured signals and the form in which the results are presented.

\section{Computational methods and theory}

\subsection{Measurement set-up}

We performed wash-out tests with the configuration shown in Fig. 1. In order to record respiratory flow and gas fractions the subject breathes via a mouth piece through a tubing system containing (1) a Fleisch No. 2 pneumotachograph (SensorMedics, Bilthoven, The Netherlands) measuring in- and expiratory flow; (2) the tip of a sample capillary connected to an Air-spec MGA3000 quadrupole mass spectrometer (Chest Scientific Instruments, Biggin Hill, U.K.); (3) a two-way valve separating in- and expiratory gas flow; and (4) a (manually controlled) three-way valve to provide inspiration gas either from room air or from a Douglas-bag containing a mixture of $21 \%$ oxygen and small fractions $( \pm 2 \%)$ of helium and $\mathrm{SF}_{6}$ in nitrogen. The mass spectrometer samples the oxygen, carbon dioxide and inert indica- 


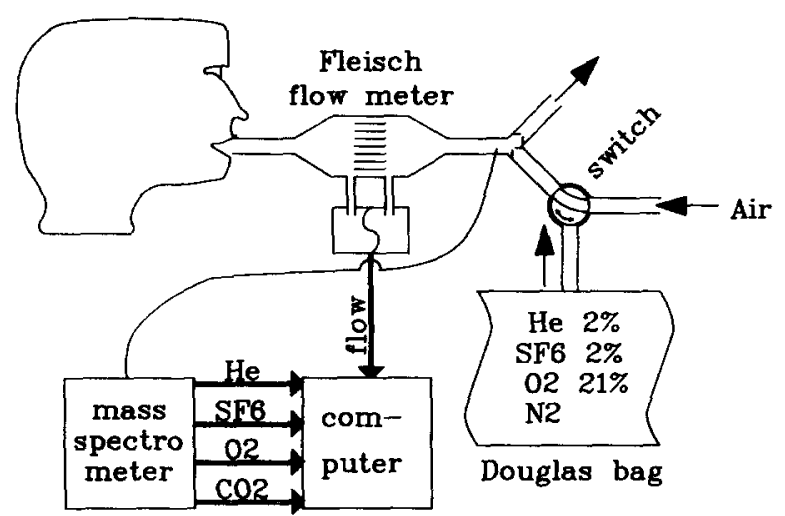

Fig. 1. Measurement set-up. Tubing system containing a Fleisch pneumotachograph, a capillary to an Airspec MGA3000 mass spectrometer, one-way valves and a gas-switch selecting gas 1 (room air) or gas 2 (from Douglas bag). The outputs of the pneumotachograph and the mass spectrometer are connected to ADC channels of the computer.

tor gas fractions in the in- and expired gas mixture with a sample frequency of $50 \mathrm{~Hz}$. The outputs of the pneumotachograph and the mass spectrometer are connected up to the A/D converter of a PDP-11 computer. First, the subject breathes from air. In order to start a wash-in test, the laboratory assistant commands the computer to start data sampling (with a frequency of $50 \mathrm{~Hz}$ ) and to store the samples on disk. One breath cycle later the laboratory assistant connects during expiration the inspiratory tube to the Douglas-bag using the switch. During the successive breath-cycles the difference between the in- and expired indicator gas fraction decreases. The duration of the wash-out is dependent on the patient. For long-healthy subjects a duration of 2 min is sufficient, but for patients with severe COPD or emphysema much more time is needed (up to $4 \mathrm{~min}$ ) to wash more than $90 \%$ of the indicator gas out of the lung. Data sampling stops automatically after a pre-set maximum time interval has elapsed or by a single-key command issued by the laboratory assistant. When the difference between the in- and expiratory gas-fraction has become negligible, a wash-out test can be initialised by switching the inspiratory gas back to air.

\subsection{Pattern recognition and analysis of the data}

After recording the wash-out test PDPS performs an off-line analysis of the obtained signals. In this analysis it discerns expirations and inspirations, finds the inspiratory indicator gas-fraction before and after the beginning of the wash-out, searches for the first wash-out inspiration and calculates characterising parameters for each expiration. The characterising parameters are for expiration number $i$ : the time it starts $\left(t_{\mathrm{b} i}\right)$, the time it ends $\left(t_{\mathrm{e} i}\right)$, tidal volume $\left(V_{\mathrm{T} i}\right)$, and for each of the metabolic and the inert indicator gases the end expiratory fraction $F_{\mathrm{E} i}$ and the flow-weighted mean expiratory fraction $F_{\mathrm{M} i}$. Both $F_{\mathrm{E} i}$ and $F_{\mathrm{M} i}$ are calculated relative to $F_{1}$, the inspiratory fraction of the gas in question. For example the flow weighted mean expiratory fraction is conventionally defined as $\frac{1}{V_{\mathrm{T}, i}} \int_{t_{\mathrm{p}, i}}^{t_{\mathrm{c}, i}} F(t) \dot{V}(t) \mathrm{d} t$, but we have chosen to define it as:

$F_{\mathrm{M}, i}=\frac{1}{V_{\mathrm{T}, i}} \int_{t_{\mathrm{b}, i}}^{t_{\mathrm{c}, i}}\left(F(t)-F_{\mathrm{I}, i}\right) \dot{V}(t) \mathrm{d} t$

$(F(t)=$ gas fraction; $\dot{V}(t)=$ flow $)$. In this way the algorithms and procedures to analyse a wash-out test are identical to those to handle a wash-in process (in which the inspiratory indicator gasfraction rises step-wise). The only difference between wash-in and wash-out is a change in sign. For convenience we further speak about wash-out although we actually mean wash-out/wash-in. Furthermore, we will assume in the rest of this article that $F_{\mathrm{I}}=0$.

\subsection{Presentation of results}

\section{Expirogram}

An expirogram is a graph showing the fractions of one or more gases in the expiratory gas mixture against expired volume or against time, during one expiration. In order to enable comparison of the expirograms of the two indicator gases (helium and $\mathrm{SF}_{6}$ ) and carbon dioxide and of subsequent expirograms of the indicator gases during the wash-out process the gas-fractions are scaled 
between the inspiratory and the end-expiratory value of the same breath. In this way the decreasing fraction of the indicator gas caused by the wash-out is only visible as an apparent increasing noise level. Fig. 2 shows an example of the first three expirograms of helium and carbon dioxide after the beginning of the wash-out of a subject with normal lungs (A) and of a subject with unequally ventilated, asynchronous emptying parts in the lungs (B). In the healthy subject the gas-fractions rise steeply to an almost level 'alveolar plateau' representing the gas of alveolar origin. The shape of the expirogram does not change in the course of the wash-out. In the recording $\mathrm{B}$ there is no sharp transition from the rising part of the curve to the alveolar plateau, while in the indicator gases the alveolar 'plateau' is very steep and steepens in the course of the wash-out, due to the fact that the regions with the highest ventilation rate empty early in the expiration and have a faster indicator gas dilution than the regions that empty later in the expiration. This phenomenon is easy to recognise by comparison of subsequent expirograms and comparison of the carbon dioxide and the helium expirogram of the same expiration.

\section{Analysis of the shape of the expirogram}

In order to obtain a single number quantification of the shape of the expirogram the fractional area of the frame in which it is drawn that is not occupied by the expirogram itself is calculated (shaded area in Fig. 3). If the $x$-axis is scaled between 0 and $V_{\mathrm{T}}$, and the $y$-axis scaled between the end-inspiratory and the end-expiratory fraction, the area in the frame itself equals to $F_{\mathrm{E}} \times \mathrm{V}_{\mathrm{T}}$ ( $F_{\mathrm{I}}$ being equal to zero). The (unshaded) area under the expirogram equals to $\int_{t_{\mathrm{h}}}^{t_{\mathrm{e}}} F(t) \dot{V}(t) \mathrm{d} t=$ $F_{\mathrm{M}} \times V_{\mathrm{T}}$, where $F_{\mathrm{M}}$ represents the flow-weighted mean expiratory gas-fraction. Now we choose the

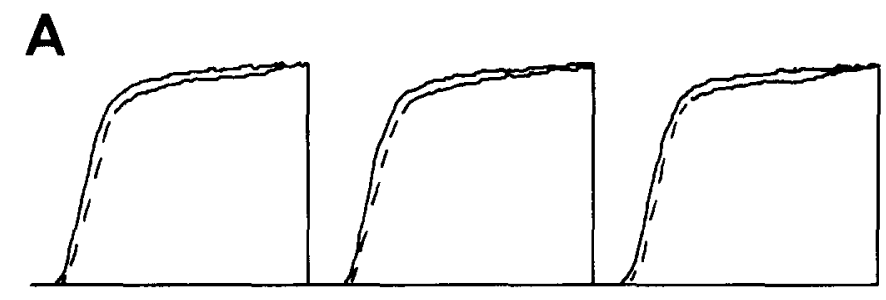

B
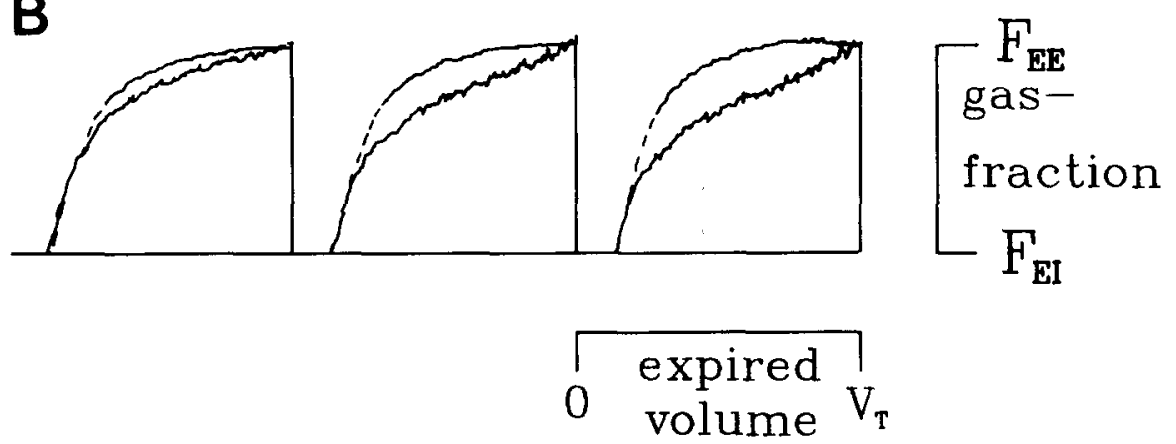

Fig. 2. The first three expirograms of $\mathrm{CO}_{2}$ (dotted line) and $\mathrm{He}$ of a subject with normal lungs (A) and of a subject with sequential emptying, unequally ventilated parts in his lungs (B). The expirogram is a single-breath graph of the expired fraction of a gas, scaled between the inspiratory and the end-expiratory indicator gas fraction, as a function of expired volume, in this figure scaled between zero and the tidal volume. The enhanced dilution of the early emptying parts of subject $\mathrm{B}$ shows up as an increasing difference between the courses of the indicator gas fraction and the $\mathrm{CO}_{2}$ gas fraction. 


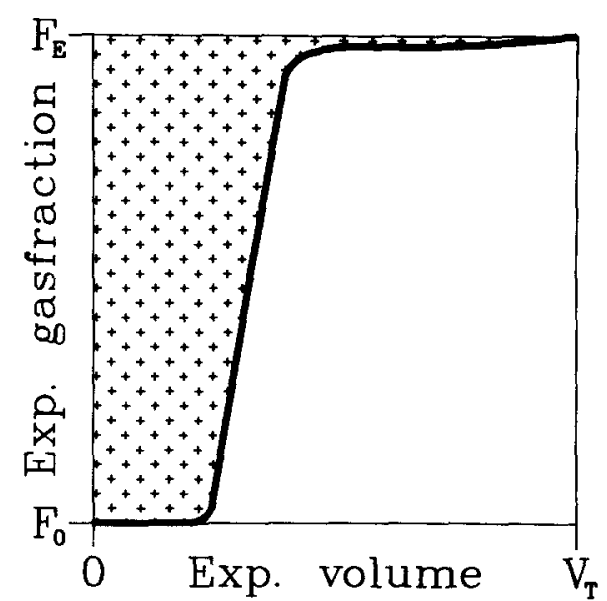

Fig. 3. Shape analysis of the expirograms. The expirogram is drawn in a normalised frame (abscissa: expired volume scaled between 0 and tidal volume; ordinate: expiratory gas fraction scaled between inspiratory fraction and end-expiratory fraction). The fraction $B$ of the area in this frame that is not covered by the expirogram (the quotient of the shaded area and the total area in the frame) serves as a single-number characteristic of the shape of the expirogram. In fact $B$ is equal to the Bohr 'dead space'.

single-number quantification of the expirogram to be the fraction $B$ of the area of the frame that is not covered by the expirogram:

$$
\begin{aligned}
B & =\frac{\text { shaded area }}{\text { total area in frame }} \\
& =\frac{F_{\mathrm{E}}-F_{\mathrm{M}}}{F_{\mathrm{E}}}
\end{aligned}
$$

This number is in fact equal to the Bohr 'dead space' fraction [18] as defined for the steady-state $\mathrm{CO}_{2}$ wash-out. Bohr 'dead space' is an approximation of the pulmonary dead space assuming (1) that a part $V_{\mathrm{D}}$ of the expired tidal volume $\left(V_{\mathrm{T}}\right)$ comes from the dead spaces containing inspiratory gas, and the rest comes from the alveolar spaces where the inspiratory gas is mixed with the gas present in the lung before the beginning of the inspiration, and (2) that the end-expiratory gas mixture is representative for the mixture in the alveolar spaces. Then the amount of each of the gases expired during a single expiration equals:

$F_{\mathrm{M}} V_{\mathrm{T}}=F_{\mathrm{l}} V_{\mathrm{D}}+F_{\mathrm{E}}\left(V_{\mathrm{T}}-V_{\mathrm{D}}\right)$

where $F_{\mathrm{M}}$ stands for mean expired gas-fraction and $F_{\mathrm{E}}$ for end-expiratory fraction. Hence, the Bohr 'dead space' fraction equals:

$$
\begin{aligned}
\frac{V_{\mathrm{D}}}{V_{\mathrm{T}}} & =\frac{F_{\mathrm{E}}-F_{\mathrm{M}}}{F_{\mathrm{E}}} \\
& =B
\end{aligned}
$$

( $F_{1}$ being equal to zero).

The value of the Bohr 'dead space' as an estimation of dead space, however, is questionable because the assumption that the end-expiratory gasfraction equals the mean alveolar fraction is generally not true. A systematic change of the breath-by-breath determined indicator gas Bohr 'dead space' value in the course of the wash-out is an indication of the existence of ventilation inhomogeneity due to sequential emptying of parallel compartments in the lung with different ventilation ratio. In order to show these systematic variations, PDPS is able to produce graphs of the subsequent 'dead spaces' for the carbon dioxide as well as for the indicator gases as a function of time. In order to reduce the influence of breath-by-breath variations of tidal volume (causing variations of the location of the diffusion front) PDPS plots a graph of the breath-by-breath differences between the Bohr 'dead space' of the indicator gas and the Bohr 'dead space' of carbon dioxide. Fig. 4 shows such a graph for the same wash-out test as used for Fig. 2. This example shows clearly how in the course of the wash-out process, the Bohr 'dead spaces' of the indicator gas increase relative to the steady-state 'dead spaces' of carbon dioxide, reflecting the changes of the expirograms.

\section{Gas exchange ratio and metabolic rate}

The gas exchange ratio $R$ is the quotient of the volume of carbon dioxide released into, and oxygen taken up per volume unit of expired gas. The program plots graphs of the gas-exchange 


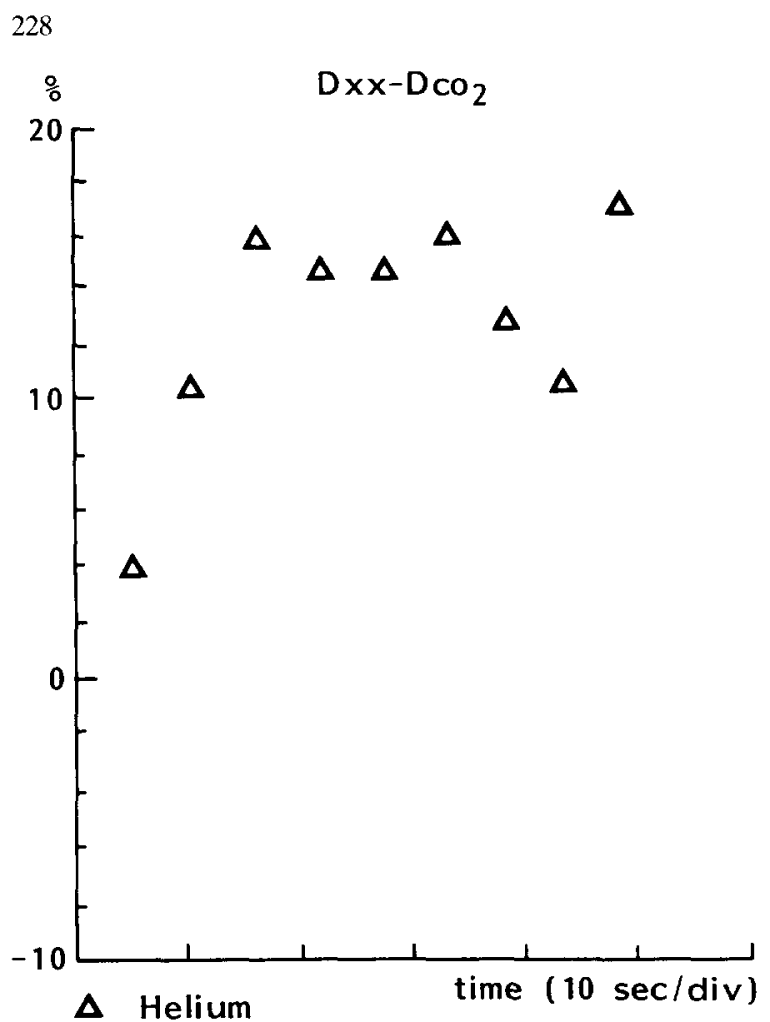

Fig. 4. Breath-by breath values of the differences of the Bohr 'dead space fraction' for He and the Bohr 'dead space fraction' for $\mathrm{CO}_{2}$. The inhomogeneity causes the 'dead space' to rise in the course of the wash-out. ratio of the expired gas as a function of expired volume. Variations in gas exchange ratio within one breath are caused by regional variations of the ventilation/perfusion ratio in the lung. In areas where ventilation is high compared to perfusion, much carbon dioxide is released compared to the amount of oxygen taken up. Fig. 5 is an example of the breath-by-breath gas-exchange ratio plots. These plots are drawn from the same breath-cycles as in Fig. 2. The course of the gas exchange ratios in part (в) shows that the ventilation inhomogeneity shown by the expirograms is not compensated by the perfusion, but that the highly ventilated parts emptying early in the expiration have a higher ventilation/perfusion ratio than the parts emptying later in the expiration.

\section{Functional Residual Capacity}

The functional residual capacity (FRC) is the volume of gas in the lung at the end of a normal expiration. The FRC at the end of the last expiration before wash-out starts can be calculated using the well-known mass-balance equation [19] stating that the partial volume in the lung occupied by the indicator gas before the wash-out, equals the sum of the partial volume of the
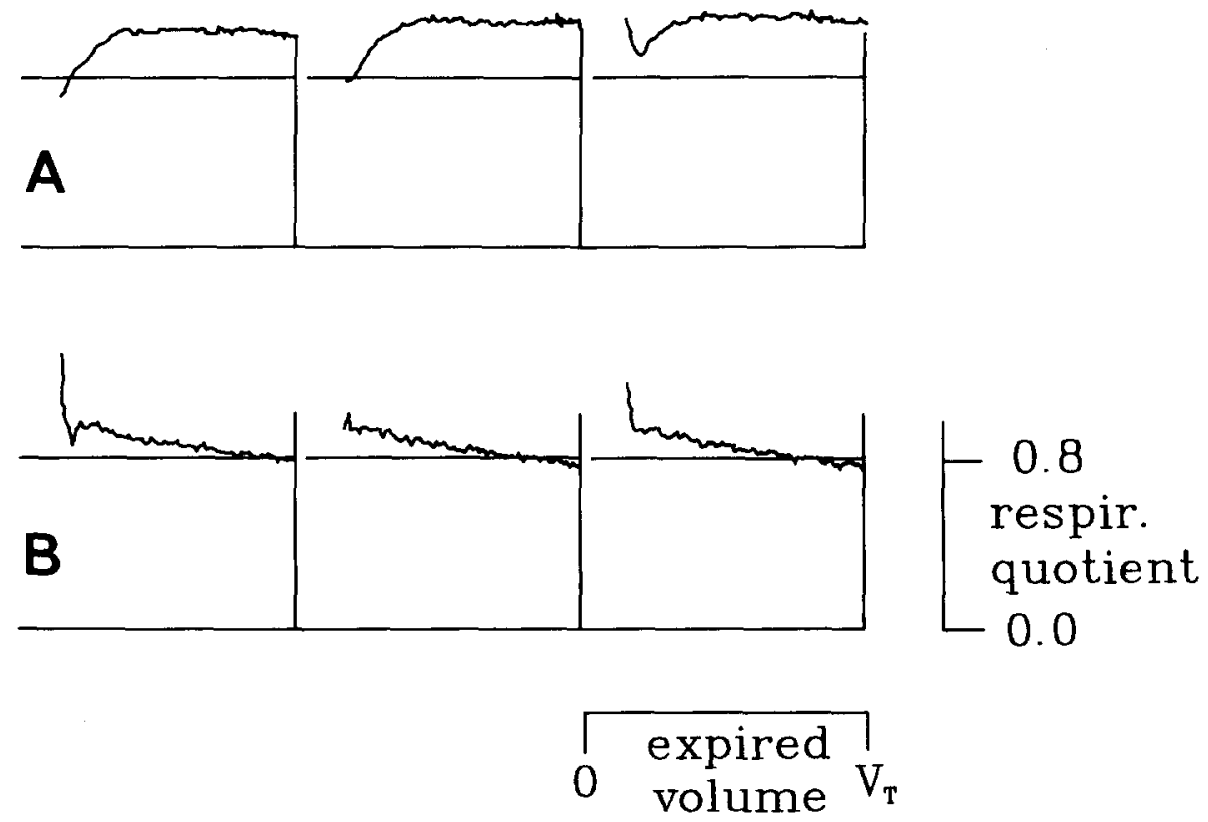

Fig. 5. Gas exchange ratio graphs (ratio of $\Delta F_{\mathrm{CO}_{2}} / \Delta F_{\mathrm{O}_{2}}$ ) of the same expirograms as in Fig. 2. The decreasing gas-exchange ratios in $\mathrm{B}$ show that the ventilation inhomogeneity is not compensated by the perfusion. 
indicator gas at the end of the $n$th wash-out expiration and the net partial volume washed out during the previous $n$ wash-out breath-cycles:

$F_{\mathrm{A} 0} \times \mathrm{FRC}=F_{\mathrm{A} n} \times \mathrm{FRC}+\sum_{i=1}^{n} F_{\mathrm{M} i} V_{\mathrm{T} i}$

$\mathrm{FRC}=\frac{\sum_{n}^{i=1} F_{\mathrm{M} i} V_{\mathrm{T} i}}{F_{\mathrm{A}} S_{0}-F_{\mathrm{A} n}}$

$F_{\mathrm{A} 0}$ and $F_{\mathrm{A} n}$ are the alveolar indicator gas fractions at respectively the end of the last expiration before wash-out and at the end of the $n$th washout expiration. Generally $F_{\mathrm{A} 0}=F_{1}$, the inspiratory indicator gas-fraction before the beginning of the wash-out. Since there is no way to measure $F_{\mathrm{A} n}$ it is approximated by the end expiratory fraction $F_{\mathrm{E} n}$. Thus, the approximation of the FRC, calculated using the first $n$ breath-cycles since the beginning of the wash-out, is equal to:

$\mathrm{FRC}_{n}^{*}=\frac{\sum_{i=1}^{n} F_{\mathrm{M} i} V_{\mathrm{T} i}}{F_{\mathrm{E} 0}-F_{\mathrm{E} n}}$

This approximation is not valid for the general case. At the end of the wash-out, when nearly all the indicator gas has disappeared, $F_{\mathrm{A} n} \approx 0$ and $F_{\mathrm{E} n} \approx 0$, and Eqn. 9 provides a good estimation of the FRC. If, for example, the wash-out is continued until $98 \%$ of the indicator gas has been washed out, and at that moment $F_{\mathrm{A} n}=2 F_{\mathrm{E} n}$, then the difference between $\mathrm{FRC}_{n}^{*}$ and the actual FRC equals $2 \%$. The accuracy can be improved by extrapolation of the curve obtained by plotting the breath-by-breath $\mathrm{FRC}_{n}^{*}$ estimations as a function of $F_{E n}$ [20].

We use the deviation of $\mathrm{FRC}_{n}^{*}$ and the actual FRC (obtained by extrapolation or a long prolongation of the wash-out) to reconstruct the deviation between $F_{\mathrm{A} n}$ and $F_{\mathrm{E} n}$ for lower values of $n$, where this deviation has a large influence on FRC ${ }^{*}$. The quotient of $\mathrm{FRC}_{n}^{*}$ and the true FRC is:

$$
\frac{\mathrm{FRC}_{n}^{*}}{\mathrm{FRC}}=\frac{F_{1}-F_{\mathrm{A} n}}{F_{1}-F_{\mathrm{E} n}}
$$

So deviations from unity of

$\frac{F_{\mathrm{A} n}}{F_{\mathrm{E} n}}=\frac{\mathrm{FRC}_{n}^{*}}{\mathrm{FRC}}+\left(1-\frac{\mathrm{FRC}_{n}^{*}}{\mathrm{FRC}}\right) \frac{F_{\mathrm{i}}}{F_{\mathrm{E} n}}$

are due to regional variations of emptying or filling rate in parts of the lung. Therefore PDPS is able to produce graphs of $F_{\mathrm{A}} / F_{\mathrm{E}}$ for the indicator gases as a function of time.

\section{The wash-out curve}

Indicator gas wash-out is described by a graph of normalised end-expiratory fraction $F_{\mathrm{E} i} / F_{\mathrm{A} 0}$ or normalised expiratory fraction $F_{n i} / F_{\mathrm{A} 0}$, as a function of time. The shape of the wash-out curve is

$P($ end $) / P($ eq $)$

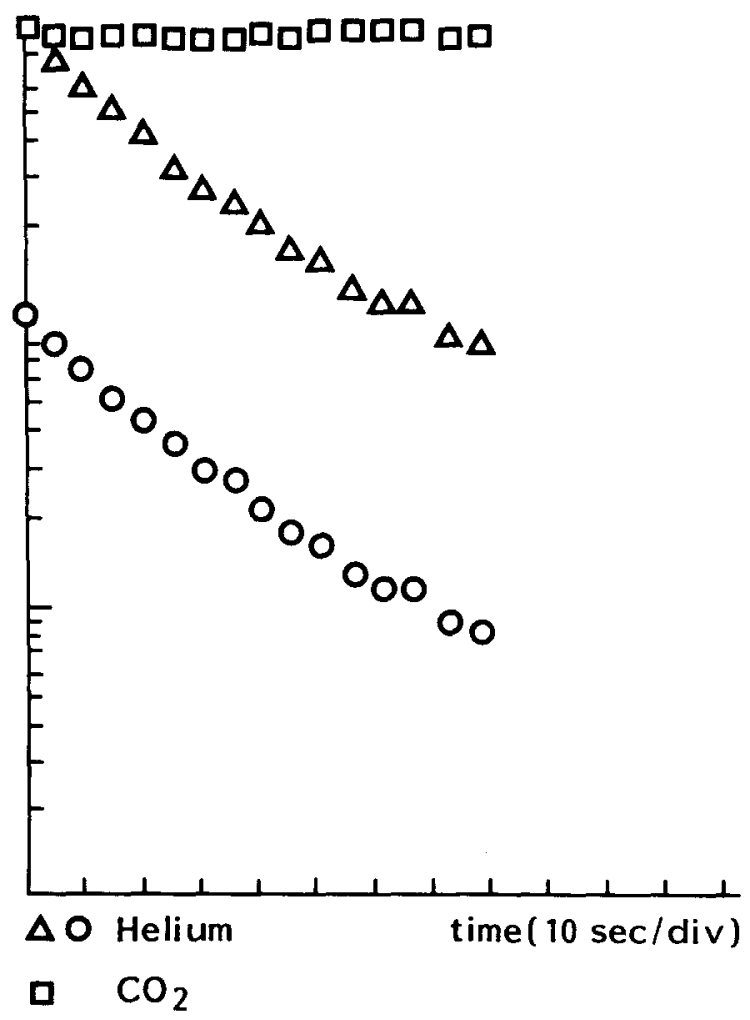

Fig. 6. Wash-out graph of $\mathrm{He}$ and $\mathrm{CO}_{2}$, showing the end-expiratory fraction of the indicator gas and $\mathrm{CO}_{2}$ as a function of time. The quotient of the $\mathrm{He}$ and $\mathrm{CO}_{2}$ values is displayed a decade lower (using circle symbols). In this way the effect of variations in tidal volume on the wash-out curve can be partly corrected. 
influenced by variations in tidal volume. To indicate these variations and to reduce their influence on the wash-out curve, the mean and endexpiratory carbon dioxide fractions are displayed as a reference, and the quotient of the indicator gas fraction and the carbon dioxide fraction is displayed a decade lower. An example is shown in Fig. 6.

\section{Program description}

For computer architectural reasons, the software system consists of a small main program performing command interpretation and a set of subprograms for specific tasks (recording, analysis and graphical output). The programs communicate by way of a chaining mechanism, and by files containing parameters or results of the preliminary analysis on data files. Command interpretation can be done in an interactive or in standalone (batch) mode. In the batch-mode a list of commands is executed on a series of data files. The command list can be stored in a file before the program starts, entered during program execution, or entered automatically by executing the commands manually while PDPs is in a 'learn mode'. An important feature is a help command, providing information about the command set.

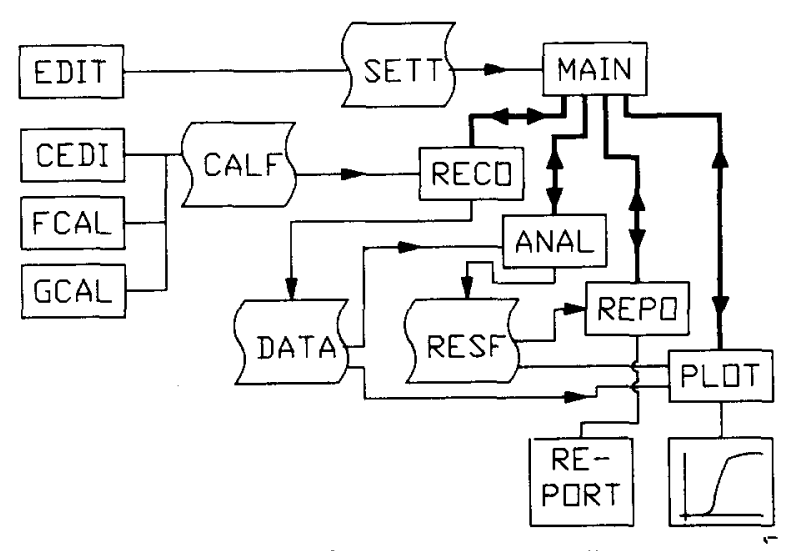

Fig. 7. Scheme of the software system. A small main program MAIN performs command interpretation and passes temporary control to other small programs. The function of the programs and files are listed in Table 1.
TABLE 1

List of the available subprograms and the files used in PDPS

\begin{tabular}{|c|c|}
\hline Program & Purpose \\
\hline MAIN & Command interpretation and control. \\
\hline RECO & $\begin{array}{l}\text { Performs data acquisition during wash-out. Writes } \\
\text { obtained samples to data file DATA }\end{array}$ \\
\hline ANAL & $\begin{array}{l}\text { Analysis of the signals in file DATA; writes calcu- } \\
\text { lated results to file RESF. }\end{array}$ \\
\hline REPO & $\begin{array}{l}\text { Reports result of analysis (read from RESF) in the } \\
\text { form of a table (Fig. } 8 \text { ) }\end{array}$ \\
\hline PLOT & Produce plots. \\
\hline CEDI & List or change calibration file CALF manually. \\
\hline GCAL & $\begin{array}{l}\text { Perform calibration of gas-fractions and write the } \\
\text { results in CALF. }\end{array}$ \\
\hline File & Purpose \\
\hline SETT & $\begin{array}{l}\text { Settings file (changable by a text editor). Contains } \\
\text { global parameter settings. }\end{array}$ \\
\hline CALF & $\begin{array}{l}\text { Contains measurement set-up (sample-frequency, } \\
\text { scheme of connections to the ADC channels) and } \\
\text { calibration data. }\end{array}$ \\
\hline DATA & $\begin{array}{l}\text { Contains the obtained samples of a wash-out ses- } \\
\text { sion in digital form. }\end{array}$ \\
\hline RESF & $\begin{array}{l}\text { Contains data obtained by analysis of the data } \\
\text { file. }\end{array}$ \\
\hline
\end{tabular}

Fig. 7 shows the structure of PDPS, and Table 1 lists the available subprograms. PDPS consists of (1) a main program, performing command interpretation from terminal or batch-file, providing helpful information to the user and starting subprograms on request, (2) a subprogram to perform data recording; (3) a subprogram to handle a file containing parameters for the record session; (4) subprograms to calibrate the gas-fraction signals or the flow signal and (5) subprograms to perform preliminary analysis on recorded data and to produce results in the form of graphs. Parameters needed for the system to run properly are stored in two files. The settings file (text file, changeable with a text editor) contains pre-set values of parameters in the program, concerning among others debugging level and mode (interactive vs. batch). It also contains the commands to be executed when in batch mode. The calibration file contains the parameters needed for the data acquisition and the calibration of the data. 


\subsection{Description of data acquisition and calibration}

Before PDPS can start a wash-out measurement, the channels of the analog-digital converter (ADC) with which the flow- and gas-fraction signals are sampled, have to be configured and the signals have to be calibrated (i.e., the values of these signals have to be mapped to the digital sampled values). In order to avoid the need to re-enter this information before each measurement, PDPS stores the following parameters in a calibration file: the sample frequency, the time delay of the gas fraction signals due to the capillary leading to the mass spectrometer, and a table. The table contains for each signal to be used an identification code (e.g., $1=$ flow, $2=$ helium, etc.), and data for a two-point calibration (a calibration point consists of the physical value of a signal (e.g., gas fraction) and the digital value to which the physical value is converted). The subprogram "GCAL" in Fig. 7 serves to calibrate gas fractions. When invoked, it continuously samples gas fractions from a gas mixture of known composition, plotting the obtained signals on the screen, until the user issues a stop command. Then the subprogram stores for each gas the mean of the last 8 samples (in order to reduce influence of noise) as the digital value of one of the calibration points (the user decides which one). The physical value is obtained from a file associated with the gas mixture used. The calibration subprogram "FCAL" serves to calibrate the flow sampling channel using a calibration cylinder with movable piston displacing a test volume of $1000 \mathrm{ml}$. The subprogram first calibrates the data corresponding to zero flow. Next, it samples the flow while the user moves the test volume in and out of the cylinder through the flow-measuring device. The volume displacement is derived from the obtained signal and is plotted on the screen to enable the user to detect nonlinearities. From the data obtained a calibration factor for expiration as well as for inspiration is calculated and displayed. The user is asked to accept one of the two values, or the mean of the two. If the flowmeasuring device is non-symmetric (it has a different calibration factor for inspiratory and expiratory flow) the user should choose the calibra- tion factor for expiratory flow. Otherwise, he should use the mean of inspiratory and expiratory flow. The subprogram calculates the digital values belonging to flow values of 0 and $1000 \mathrm{ml} / \mathrm{s}$, and stores them in the recording description file.

\subsection{Data acquisition}

The data acquisition subprogram asks the user for the maximum duration of the measurement, the name of the file to store the data, and comments to be included in this data file. Recording is started by manual (one-key) command or external triggering. The signals are sampled in a swept mode, and the samples are stored, unaltered, in the data file. After the measurement time has been elapsed or the user has interrupted the subprogram by a key, sampling stops and a trailer block containing the information from the calibration file and the user comments is appended to the file. After the measurement the computer plots the acquired signals on the screen.

\subsection{Analysis of the signals}

The analysis subprogram performs the detection of the expirations and performs the preliminary analysis (as described in a previous section). The results are written in a file, and are used by other subprograms.

\subsection{Presentation of the results}

The plotting subprogram draws plots on the terminal, with a hardcopy option. The following plots can be made.

(a) Breath-by-breath expirograms of one or more of the gases carbon dioxide, helium or $\mathrm{SF}_{6}$ (Fig. 2). Expirograms of one breath are drawn in the same frame. For ordinate, a choice can be made between relative volume $\left(0 \ldots V_{\mathrm{T} i}\right)$, absolute volume $(0 \ldots 500 \mathrm{ml}$, in order to compare phase II of expirograms with different tidal volume) or time $\left(t_{\mathrm{b} i} \ldots t_{\mathrm{e} i}\right)$. The $y$-axis is normalised (scaled between the end-inspiratory and end-expiratory fraction) for each of the gases separately. The subprogram plots by default 15 subsequent ex- 
pirograms on the same screen (three rows of 5 frames).

(b) Gas exchange ratio of each subsequent expiration as a function of time, relative or absolute volume (Fig. 5). Optionally, expirograms of carbon dioxide and oxygen are included.

(c) Dead space: To analyse variations of the shape of the expirograms the differences of the dead space of the chosen gases and the dead space of carbon dioxide of the same breath is plotted breath-by-breath as a function of time (Fig. 4);

(d) Wash-out curve (Fig. 6). The carbon dioxide corrected wash-out curve is plotted a decade below the actual curve;

(e) $F_{\mathrm{A} n} / F_{\mathrm{E} n}$ as a function of time. In addition to the plots, the report subprogram can print a table containing a breath-by-breath list of the following quantities: time; tidal volume; for each of the gases oxygen, carbon dioxide, helium and $\mathrm{SF}_{6}$ the end expiratory fraction, the mean expiratory fraction, and Bohr 'dead space'; carbon dioxide production, oxygen consumption and gas exchange ratio. Fig. 8 is an example of such a table.

\section{Hardware and software specification}

The software is written in Fortran-77 to run on DEC PDP-11 computers. There is a version running under RT11, and a version for RSX-11M-PLus. The software supports a programmable clock, and analog-digital converters. Graphical output is presented on graphical terminals of the DEC VT series. The pictures can be captured by the com-

$\begin{array}{lllllllllllllllll}G & A & S & T & R & A & N & S & P & O & R & T\end{array}$

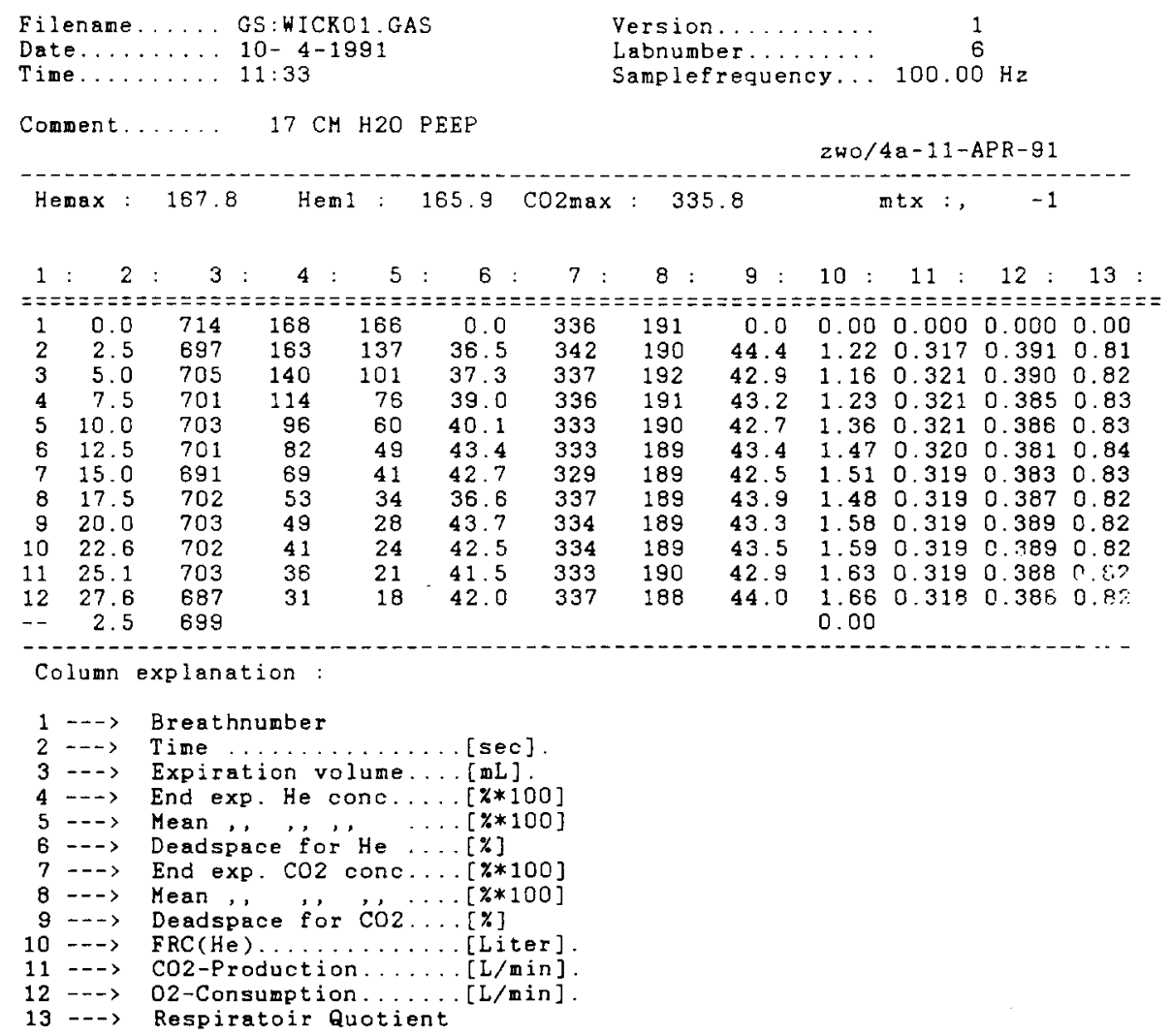

Fig. 8. Table containing breath-by-breath results. This table contains tidal volume, mean- and end expiratory fractions and Bohr 'dead space fractions' of $\mathrm{He}$ and $\mathrm{CO}_{2}$, FRC derived from $\mathrm{He}$ wash-out and gas-exchange data $\left(\mathrm{O}_{2}\right.$ uptake, $\mathrm{CO}_{2}$ delivery and respiratory quotient). 
puter to make hardcopies on REGIS-compatible printers. Currently, we are converting the program in order to run it on IBM PC-compatible computers under MS-DOS.

\section{Discussion}

PDPS is a software system that has as its purpose gaining insight in the distribution of ventilation and ventilation/perfusion ratio in the lung. As far as we know, this is the first system that uses the advantage of the capacity of a mass spectrometer to measure simultaneously the fractions of the metabolic gases (oxygen and carbon dioxide) and of blood-insoluble indicator gases washing out of the lung. This type of measurement enables combination of several calculations on data obtained during the same train of breath-cycles. Besides established parameters being calculated we introduce new methods (the reconstruction of the ratio of the mean alveolar to end-expiratory indicator gas-fraction, and the breath-by-breath Bohr dead-space analysis as indication of a changing shape of the expirogram) as a means of evaluation the efficacy of ventilation. Combining information from the various calculations leads to a more comprehensive description of the ventilation and the ventilation/ perfusion process than the individual methods alone would permit. For example, in patients with obstructive pulmonary disease or bronchus carcinoma the lungs can be divided in compartments with different ventilation ratios $(\dot{V} / V)$, that empty asynchronously (best ventilated compartments empty at the beginning of the expiration) and where the ventilation inhomogeneity is not compensated by complementary perfusion inhomogeneity. In that case, the ventilation inhomogeneity shows up in multi-exponential decay of the expiratory indicator gas-fraction and the ventilation/perfusion mismatch shows up in a sloping alveolar plateau of the carbon dioxide expirograms and a gas-exchange ratio that diminishes in the course of a single expiration. Furthermore, the asynchronous emptying of the parallel compartments causes the Bohr 'dead space' fractions of the subsequent expirograms during the wash-out process to rise. These phenomena are illustrated in Figs. 2B, 4 and 5B. However, in many cases wash-out tests show multi-exponential wash-out and a sloping alveolar plateau of carbon dioxide, but the gas-exchange ratio does not change in the course of a single expiration and the Bohr 'dead space' fraction does not change significantly in the course of the wash-out [12]. In that case, the ventilation inhomogeneity can not be caused by unequally ventilated, asynchronously emptying volumes, but it must be caused by a stable longitudinal gas-fraction gradient at the level of the respiratory bronchioli where the gas-transport is dominantly caused by diffusion [10]. It is only by the combination of the techniques that this difference can be recognised.

An alternative way to study the diffusion-related ventilation inhomogeneity is offered by PDPS allowing the combination of the washout of two gases with different diffusion coefficient, like helium and $\mathrm{SF}_{6}$ (the former being 6-times more diffusible than the latter). The origin of diffusion-related inhomogeneity is not yet fully understood. Von Nieding et al. [21] performed an extensive study on $\mathrm{He} / \mathrm{SF}_{6}$ washout and found that $F_{\mathrm{E}} / F_{0}$ of helium was larger than $F_{\mathrm{E}} / F_{0}$ of $\mathrm{SF}_{6}$ at the beginning of the wash-out, but smaller at the end of wash-out. In healthy volunteers and bronchitis patients the moment of cross-over was much earlier than in emphysema patients. The delayed cross-over was ascribed to gross dilation of the airways of the 17-18 generation causing the inspiratory gas to penetrate less far into the lung than in a normal lung. Luijendijk et al. [22] showed by mathematical simulation that existence of small closed volumes in the acini, in which gas exchange takes place by collateral ventilation, could explain the cross-over retardation. These studies strongly suggest that diffusion can be a limiting factor in the gas transport in the lung, and this can be studied only with wash-out techniques using gases with different diffusability.

Although not described in this paper, the analysis of the data is complete enough to calculate conventional indices of ventilation inhomogeneity from the wash-out data (like Becklake index, moment ratio index etc. [1,2]). Using the software system described we have recently developed a 
new ventilation inhomogeneity index [20] capable of identifying COPD patients. The accuracy of the mass spectrometer enables the addition of only small fractions of the indicator gases $(2 \%)$ into the inspiratory gas-mixture during wash-in. This has the advantages that no secondary gas effect occurs, that the inspiratory oxygen fraction can be kept constant, so that metabolic measurements can be performed during the wash-out, and that the wash-out can be performed on critically ill patients needing very high inspiratory oxygen levels. PDPs enables calculations to be made for wash-in as well as wash-out tests. This has the advantage of utilising the time preceding washout, that is needed to wash the indicator gas into the lungs, by performing a measurement. This results in an optimal use of measurement time which can be advantageous in the rapidly changing condition of the critically ill patient. The obtained data, besides providing patient lungfunction tests $[12,23,24]$, can be used for basic lung-physiological research. With the described system measurements have been performed on 60 spontaneously breathing patients. Measurements can be performed using a special device to inject the indicator gas into the inspired gas mixture [23]. We performed wash-out tests on 65 mechanically ventilated Intensive Care patients in order to evaluate the effect of ventilatory settings (e.g., application of positive end-expiratory pressure ventilation) and medication (e.g., bronchodilators) on the end-expiratory lung-volume and ventilation inhomogeneity [24].

\section{Mode of availability}

The program sources are available on tape or MS-Dos diskette. Requests should be addressed to the author.

\section{Acknowledgement}

This investigation was sponsored by the Dutch organisation for pure research, zwo, and in part by the Netherlands Asthma Foundation. The au- thors thank W.P.J. Holland, MSc., and Dr. C. Ince for careful reading of the manuscript.

\section{References}

[1] G.J. Gallivan and W.N. McDonell, An evaluation of the multiple-breath nitrogen washout as a pulmonary function test in dairy cattle. Can. J. Vet. Res., 53 (1989) 133-142.

[2] G.M. Fleming, E.H. Chester, J. Saniie and G.M. Saidel, Ventilation inhomogeneity using multi-breath washout; comparison of moment ratios with other indexes. Am. Rev. Respir. Dis., 121 (1980) 789-794.

[3] W.S. Fowler, E.R. Jr. Cornish and S.S. Kety, Lung function studies. VIII. analysis of alveolar ventilation by pulmonary $\mathrm{N}_{2}$ clearance curves. J. Clin. Invest., 31 (1952) 40-50.

[4] T. Hashimoto, A.C. Young and C.J. Martin, Compartmental analysis of the distribution of gas in the lung. J. Appl. Physiol., 23(2) (1967) 203-209.

[5] B.A. Sjöqvist, K. Sandberg, O. Hjalmarsson and D. Olsson, Method for analysing multiple-breath nitrogen wash-outs. Med. Biol. Eng. Comput. 24 (1986) 83-90.

[6] D.M. Gomez, W.A. Briscoe and G. Cumming, Continuous distribution of specific tidal volume throughout the lung. J. Appl. Physiol., 19(4) (1964) 683-692.

[7] G. Lamedica, V. Brusasco, A. Taino and R. Ramonio, Analysis of nitrogen multibreath washout curves through a statistical approach. Respiration, 39 (1980) 333-343.

[8] S.M. Lewis, J.W. Evans and A.A. Jalowayski, Continuous distribution of specific ventilation recovered from indicator gas washout. J. Appl. Physiol., 44 (1978) 416-423.

[9] T. Nakamura, T. Takishima, T. Okubo, T. Sasaki and H. Takahashi, Distribution function of the clearance time constant in lungs. J. Appl. Physiol., 21 (1966) 227-232.

[10] L. Engel, Gas mixing within the acinus of the lung. $J$. Appl. Physiol., 54(3) (1983) 609-618.

[11] W.R. de Vries, S.C.M. Luijendijk and A. Zwart, Helium and sulfurhexafluoride washout in asymmetric lung models. J. Appl. Physiol., 51(5) (1981) 1122-1130.

[12] A. Zwart, Ventilation and perfusion distributions in lungs obtained by mass spectrometry. In Proceedings of the IEEE Engineering in Medicine \& Biology Society 10th Annual International Conference, pp. 820-822, 1988.

[13] S.C.M. Luijendijk, A. Zwart, W.R. de Vries and W.M. Salet, The sloping alveolar plateau at synchronous ventilation. Pflügers Archiv., 384 (1980) 267-277.

[14] W.S. Fowler, Lung function studies II. The respiratory dead space. Am. J. Physiol., 154 (1948) 405-416.

[15] S. Tsunoda, A.C. Young and C.J. Martin, Emptying patterns of lung compartments in normal man. J. Appl. Physiol., 32 (1972) 644-649.

[16] R. Serra and B.F. Visser, Diagramme $\mathrm{O}_{2}-\mathrm{CO}_{2}$ alveolaire, Poumon Coeur, 10 (1963) 1261-1272. 
[17] H. Rahn and L.E. Fahri, Ventilation, perfusion and gas exchange - the $\dot{V}_{\mathrm{A}} / \dot{Q}$ concept. in eds. W.O. Fenn and H. Rahn, Handbook of Physiology. Volume I: Respiration, Ch. 30, pp. 735-766, (Am Physiol Soc, Washington, DC, 1964).

[18] J.S. Haldane and J.G. Priestley, The regulation of the lung ventilation. J. Physiol., 32 (1905) 225-266.

[19] C.R. Felton, H.D. Montenegro and G.M. Saidel, Inspiratory flow effects on mechanically ventilated patients: lung volume, inhomogeneity, and arterial oxygenation. Intensive Care Med., 10 (1984) 281-286.

[20] P.E.M. Huygen, H. Strijdhorst, C. Ince, A. Zwart, B.W.A. Feenstra and H.A. Bruining, A new measure for pulmonary ventilation inhomogeneity using indicator gas washout test. Pflügers Arch, 414 (1989) S198.

[21] G. von Nieding, H. Löllgen, U. Smidt and H. Linde,
Simultaneous washout of helium and sulfur hexafluoride in healthy subjects and patients with chronic bronchitis, bronchial asthma, and emphysema. Am. Rev. Respir. Dis., 116 (1977) 649-660.

[22] S.C.M. Luijendijk, W.R. de Vries, and A. Zwart, Collateral ventilation by diffusion and the exchange of inert gases in the lung. Eur. Resp. J., 3 (Suppl. 10) (1990) 186 s.

[23] P.E.M. Huygen, B.W.A. Feenstra, W.P.J. Holland, C. Ince, H. Stam and H.A. Bruining, Design and validation of an indicator gas injector for multiple gas wash-out tests in mechanically ventilated patients. Crit. Care Med., 18 (1990) 754-759.

[24] I. Gültuna, P.E.M. Huygen, H. Strijdhorst, C. Ince and H.A. Bruining, Clinical applications of an indicator washout test during artificial ventilation, Intensive Care Med., 16 (1990) 165. 